\title{
USING OF ENDOVASCULAR CATHETER METHODS IN SURGICAL TREATMENT PATIENTS WITH LUNG BLEEDING
}

DOI: 10.36740/WLek202006113

\author{
Kateryna V. Ponomarova' ${ }^{1}$ Dmytry V. Minukhin², Denis 0.Yevtushenko², Andrey V. Tokarev², \\ Oleksandr M. Kudrevych ${ }^{3}$, Vasiliy G. Hroma² \\ 'SI V.T. ZAYTSEV INSTITUTE OF GENERAL AND EMERGENCY SURGERY OF NAMS OF UKRAINE, KHARKIV, UKRAINE \\ 2KHARKIV NATIONAL MEDICAL UNIVERSITY, KHARKIV, UKRAINE \\ 3V. N. KARAZIN KHARKIV NATIONAL UNIVERSITY, KHARKIV, UKRAINE
}

\begin{abstract}
The aim: Improve the results of the treatment of patients with pulmonary bleeding.

Materials and methods: We examined 57patients with lung bleeding different etiology, who were hospitalized in the department of Thoraco-Abdominal Surgery. All patients were divided into two groups - of the main group 27patients and the comparison group 30 patients, depend of ages, sex, nosological form, level of lung bleeding. Patients aged from 27 to 78 years, including 34 men (62,5\%) and 23 women (37,5\%.). The test diseases includes: bronchiectasis disease - in 21 (37,1\%), pulmonary fibrosis with malformation $\mathrm{BA}$ - in $14(24,7 \%)$, abscess of the lung - in $9(15,9 \%)$, polycystic lung disease - in $6(12,7 \%)$, chronic obstructive pulmonary disease - in 5 (9,6 \%).

Results: As a result of complete physical examination of patients with LB, it has been established that hemorrhage was the result of obstructive bronchitis in 14 patients (42\%), there was chronic obstructive pulmonary disease in 7 (21\%) and bronchiectasis was diagnosed in 6 (18\%) patients. In 2 (6\%) patients pulmonary hemorrhage was caused by community-acquired pneumonia. Central lung cancer was detected in 4 (12\%) patients.

Conclusions: Bronchial artery angiography gives high efficiency in solving the problem of hemostasis in oncological and nonspecific lung diseases. Endovascular occlusion of bronchial arteries permits: to elaborate diagnosis because of the presence of specific angiographic signs of malignant tumor; to perform effective endovascular hemostasis.
\end{abstract}

KEY WORDS: embolization of bronchial arteries, hemostasis, pulmonary bleeding

Wiad Lek. 2020;73(6):1149-1153

\section{INTRODUCTION}

Treatment of pulmonary bleeding is still one of the most pressing problems in surgery. Pulmonary bleeding is the most dangerous complication of a number of diseases [1,2]. There are 40 nosological forms that can be complicated by pulmonary hemorrhage $(\mathrm{PH})$, some authors describe $\mathrm{PH}$ as a complication in more than 100 diseases $[3,4]$. Over the last decade, the role of obstructive and purulent pulmonary diseases, which significantly suppressed pulmonary tuberculosis, has in creased in the emergence of LB $[5,6]$.

Pulmonary bleeding is often a complication of various respiratory system diseases that are associated with the inflammatory process, which are found in $8-17 \%$ of hospitalized patients [7, 8]. Most authors believe that in 95-98\% of observations the source of pulmonary bleeding in patients is bronchial arteries, which depart from the aorta and pleura $[9,10]$. A considerable amount of surgical remedies to combat this threatening complication, often complicates the choice of optimal treatment tactics and leads to the expansion of indications for the further implementation of surgical interventions, which may adversely affect the expected results of treatment and rehabilitation of patients in the early and long periods. [11]. Despite certain advanc- es in surgical treatment of pulmonary hemorrhage, which is associated with the use in the treatment of endovascular technologies, the mortality rate is still uitehigh, according to various authors, ranges from $15,1 \%$ to $57,0 \%[12,13]$.

To date, $\mathrm{X}$-ray vascular occlusion (REO) of the bronchial arteries allows patients to avoid surgery at the height of bleeding. Despite the fact that this method has been used in clinical practice for more than 30 years, the questions concerning the tactics of interventions and the choice of embolizing materials $[14,15]$. Efficiency of the method in the long-term period is devoted to the publications. In particular, in young patients, pulmonary hemorrhage may be the first sign of the disease, while the lungs may lack morphological changes in the lung tissue. [16, 17].

Currently, a large number of researchers believe that urgent surgery should be performed at the heigh to $\mathrm{PB}$ in the absence of the effect of conservative treatment. X-ray vascular occlusion (REO) of the bronchial arteries allows patients to avoid surgery at the heigh to bleeding [18]. A significant addition to the arsenal of methods of surgical treatment of pulmonary hemorrhagewas made by intervention radiology. Among these treatments, traumatic X-ray and vascular interventions are becoming increasingly important [19]. 
The use of the achievements of interventional radiology and the use of modern endovascular catheter technologies in surgeryin the late XX - early XXI centuries allowed to introduce a more advanced approach to the diagnosis and treatment of pulmonary bleeding [20]. Based on the level of development of interventional radiology in Ukraine, X-ray and vascular the rapies for the treatment of pulmonary hemorrhages of different etiology have not yet become widespread due tothe lack of angiographic equipment in most clinics to provide highly qualified X-ray surgical care. The aim of the study. Improving the treatment of patients with pulmonary hemorrhage through widespread use of hemostasis for endovascular surgery [21].

\section{THE AIM}

The aim of research - improve the results of the treatment of patients with pulmonary bleeding through widespread use of endovascular surgery methods for hemostasis.

\section{MATERIALS AND METHODS}

The study was conducted in the department of Thoraco-Abdominal Surgery V.T. Zaytsev Institute of General and Emergency Surgery of NAMS of Ukraine. We examined 57patients with lung bleeding different etiology, who were hospitalized in the department of Thoraco-Abdominal Surgery. All patients were divided into two groups - of the main group 27patients and the comparison group 30 patients, depend of ages, sex, nosological form, level of lung bleeding. Patients aged from 27 to 78 years, including 34 men $(62,5 \%)$ and 23 women $(37,5 \%$.). The test diseases includes: bronchiectasis disease - in $21(37,1 \%)$, pulmonary fibrosis with malformation BA - in $14(24,7 \%)$, abscess of the lung - in $9(15,9 \%)$, polycystic lung disease - in $6(12,7 \%)$, chronic obstructive pulmonary disease - in $5(9,6 \%)$.

Roentgen-endovascular embolization of bronchial arteries was performed using and Phillips IntegrisAllura12C (Holland). Bronchial artery angiography consists of the following: femoral cateterization is done by Seldinger technique under topical anesthesia $(0,25 \%$ solution of novocaine). Cateterization is performed by Cobra catheter with flexure $45-65$ degrees. Catheter was inserted at T4-T5 level under control of monitor and then it is fixed in the bronchial artery or intercostal artery mouth by rotational movement of guide. $1 \mathrm{ml}$ of contrast agent is added for monitoring of catheter location in required vessel. Polyurethane emboli having diameters of $450-720$ mmwere used for embolization. Emboli in combination with microembols were inserted at a big diameter of pathologically changed vessel that enhances effect of embolization. Absence of inflow of contrast agent and enhancement of distal part of bronchial arteries were considered as criteria of effective embolization.

Statistical comparisons were conducted using Student's $\mathrm{t}$-test statistically significant at $\mathrm{p}<0.05$. Fisher's angular transformation. The calculations were performed using software STATISTICA for Windows and Microsoft Excel 10.0.

\section{RESULTS}

Immediate positive full effect of endovascular embolization bronchial artery in the form of a complete stop bleeding and was observed in $46(80,8 \% \pm 1,2, \mathrm{p} \leq 0,05)$ of patients. Similar efficiency endovascular hemostasis of the other authors also perceive. Late relapses of lung bleeding and blood coughing was observed in $12(20 \% \pm 2,1, \mathrm{p} \leq 0,05)$ of patients.

Treatment of patients was started from the hemostatic therapy: $5 \%$ solution of EACA, solution of ethamsylati, dicynoni, vicasoil, tranexamic acid. In patients with pulmonary hemorrhage of II grade performed hemostatic therapy was added with introduction of albumin solution, fresh frozen plasma. Packed red cell transfusion was prescribed patients with pulmonary hemorrhage of III severity. Pulmonary hemorrhage was stopped in $17(29,8 \% \pm 2,9, \mathrm{p} \leq 0,001)$ with the use of roentgen-endovascular hemostasis of bronchial arteries. High efficiency of roentgen-endovascular occlusion in pulmonary hemorrhage of different etiology was noted by majority of authors. Recurrence of bleeding in the long period after REO appeared in $2(3,5 \% \pm 1,1, \mathrm{p} \leq 0,001)$ inoperable patients with bronchiectasis disease.

Classification of pulmonary hemorrhages proposed by Grigoryev E.G., 19831 grade - hemoptysis: 1a $-50 \mathrm{ml}$ per day; $1 \mathrm{~b}-50-200 \mathrm{ml}$ per day; $1 \mathrm{c}-200-500 \mathrm{ml}$ per day. 2 grade - massive pulmonary hemorrhage: $2 a-30-200$ $\mathrm{ml}$ per hour; $2 \mathrm{~b}-200-500 \mathrm{ml}$ per hour. 3 grade - profuse pulmonary hemorrhage: $3 a-100 \mathrm{ml}$ per saltum; $3 \mathrm{~b}-$ more than $100 \mathrm{ml}$ per saltum + obstruction of tracheobronchial tree, asphyxia. Classification depending on the extent of blood loss: 1. minor pulmonary hemorrhage - from 50 to $100 \mathrm{ml}$ per day; middle pulmonary hemorrhage - from 100 to $500 \mathrm{ml}$ per day; 2 . severe pulmonary hemorrhage - more than $500 \mathrm{ml}$ per day; 3. extra severe, profuse pulmonary hemorrhage - more than $500 \mathrm{ml}$ per day; 4. bleeding arising per saltum or for a short period.

Small LB and blood coughing was detected in 15 (26,3\% $\pm 2,2, p \leq 0,001)$ of patients, medium and massive with highlight more than $200 \mathrm{ml}$ of blood - in $9(15,9 \% \pm 1,8$, $\mathrm{p} \leq 0,001)$. Full treatment after REE of bronchial arteries radical no operation was possible in $1(1,7 \%)$ patient. Required complex examination of patients turned on a computer tomography of organs of the chest cavity and bronchofibroscopy. The use of these methods allowed pain most observations set side of defeat.

Upon identification pathologically effected BA (bronchial artery), by exercising neck their blood supply, in BA to the conductor as distally as possible a working catheter was inserted. A catheter was inserted through polyurethane emboli with a diameter 400 - 750 microns. Embolysis control on was carried out by contrast BA, revealed its stump and 40 lack of admission contrast substance in the distal sections. With a pathological change in BA, increase in diameter, availability malformations polyurethane embolis, gelatineembolis combined with the introduction of microspirals whose use allowed to reliably close the extended BA test. With the powered by lung parenchyma view and the presence of clots in the respirator paths after 


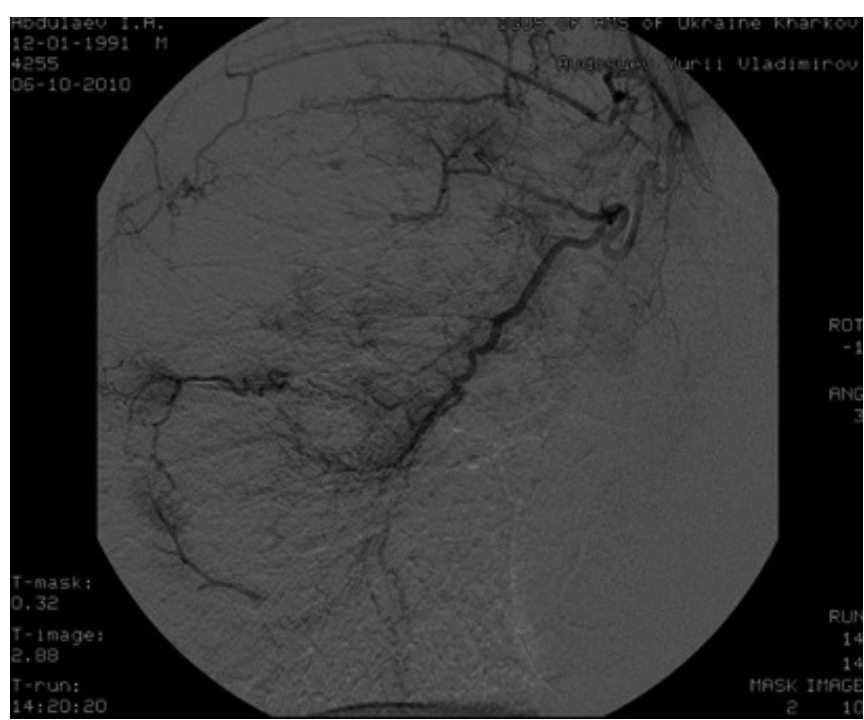

Fig. 1. Angiography of patient with bronchiectatic disease complicated by lung bleeding from bronchial artery before embolization of bronchial arteries.

roentgen-endovascular embolization of bronchial arteries carried out rehabilitation bronchofibroscopy combined with a short course antibacterial therapy and prescribe drugs to relieve pain.

\section{DISCUSSION}

The materials used by the department of Thoraco- Abdominal Surgery the State Institution «Institute of General and Emergency Surgery. V.T. Zaitsev NAMS of Ukraine. In accordance with the set tasks, complex clinical and laboratory, instrumental examination and treatment of 57 patients with pulmonary hemorrhages of different etiology were performed. When analyzing the data obtained from the examination and treatment of patients in the main group and the comparison group, it can be noted that properly organized conservative treatment of pulmonary hemorrhage using minimally invasive technology is, in most cases, quite effective and avoids severe, sometimes lethal, complications that are almost are inevitable for surgery performed at the height of pulmonary hemorrhages. Our studies have allowed us to determine the following indications for X-ray vascular occlusion in pulmonary hemorrhage: 1 . Pulmonary bleeding in inoperable patients with inflammatory diseases of the lungs and bronchi. 2. Pulmonary hemorrhage in operable patients to stabilize the patient, in-depth clinical examination and reduce operative blood loss. 3. Pulmonary hemorrhage in limited fibrotic processes, clinical manifestations of which are expressed only by hemorrhage, and resection surgery is ineffective because of the possible risk. 4. Prolonged postoperative intra-pleural bleeding with undetected source localization, recurrent hemorrhages from operated lungs to preserve lung function and to prevent recurrent operations.

The absolute contraindications to the performance of radiographic occlusion were considered: cerebral coma, severe degree of hepatic-renal failure, unreliable catheter

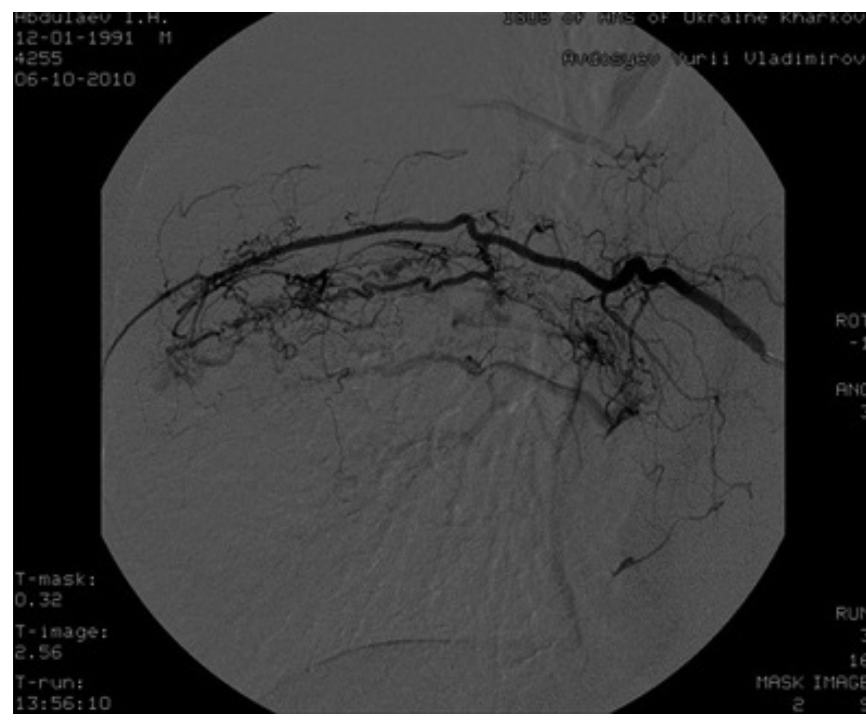

Fig. 2. Angiography of patient with bronchiectatic disease complicated by lung bleeding from bronchial artery after embolization of bronchial arteries.

fixation in the vessel, incompatibility of the occlusive substances and anatomical features of the vessels to be excluded. The relative contraindications were: pronounced intolerance of iodine-containing drugs, doubtful REO at high risk of intervention, age of the patient.

\section{CASE PRESENTATION}

We present the case of a 72-year-old man, was admitted in department of Thoraco- Abdominal surgery of ST V.T. Zaytsev Institute of General and Emergency Surgery of NAMS of Ukraine. He had been medicated for haemophtysis with more $80 \mathrm{ml}$ twice daily since 2 months ago. Anamnesis vitae - non-smoker, retired from a cran master. He presented after 8 days of haemoptic productive cough, bilateral posterior thoracic pain, fever and fatigue.Physical examination showed pale skin, afebrile and tachypnea of 20 cycles per min. Laboratory studies showed anaemia with decrease haemoglobin of $90 \mathrm{mmol} / \mathrm{L}$, normal white cell and platelet count; elevated C reactive protein $23 \mathrm{mg} / \mathrm{L}$. On the first day of hospitalisation, clinical features occurred with dyspnoea and an episode of haemoptysis. The diagnosis of bronchiectatic disease complicated by lung bleeding from bronchial artery was stand after angiography. Flexible bronchoscopy with bronchoalveolar lavage (BAL) was performed 2 days after admitted in clinic. Bacteriological revealed no endobronchial lesions or active bleeding. Bacteriological cultures were negative for bacteria, mycobacteria and fungi. Cytology was negative for malignant cells. The patient underwent conservative hemostatic therapy for the first 3 days after hospitalization. In connection, with the increase in pulmonary hemorrhage, the patient perform minimally invasive intervention in the form of embolization of bronchial arteries.

45 patients with pulmonary hemorrhages, $16(28,1 \%)$ were treated only conservatively. One of the main components of conservative treatment of pulmonary hemorrhage 
in patients of the main groupwas artificial controlled hypotension, whose main purpose was to reduce the initial blood pressure by $90-60 \mathrm{~mm} \mathrm{Hg}$ century, which in turn reduced the intensity of blood loss and increased the effect of hemostatic drugs.

Thus, the majority of patients admitted to the clinic with different types of pulmonary hemorrhages were cured by a complex of conservative measures without resorting to surgical methods of treatment. This group consisted mainly of patients with small short-term bleeding, which was not accompanied by a decrease in blood pressure and a decrease in the amount ofhemoglobin (pulmonary bleeding I - II severity).

Our research suggests that bronchoscopic examination of patients with pulmonary hemorrhages is one of the leading and reliable diagnostic methods. The results of diagnostic and therapeutic bronchoscopies in 53 patients indicate the safety and high informative content of this study.

In our opinion, endobronchial occlusion is an important step in the preoperative preparation of the patient. Due to the filling of the bronchus, it is possible to achieve a temporary discontinuation of the LB, which makes it possible to fully examine the patient, to determine the cause and source of bleeding, to correct disorders caused by the main disease and blood loss, to choose the optimal timing and type of further surgical treatment. Bronchial occlusion in our observations was performed by $12(19.1 \%)$ patients and lasted from 5 to 7 days, the maximum term -9 days. We did not observe any complications in the form of exacerbation of the inflammatory process, respiratory failure or migration of the filling of the obturator in the airways. After removal of a filling in a bronchus local changes of a mucous in the form of its hyperemia and edema, which were inverse character, were noted.In all $12(19.1 \%)$ cases, when the patient's condition allowed to perform surgery, this technique made it possible. The study was conducted in 34 (53.9\%) patients, with 32 (50.8\%) patients undergoing bronchial arteriography and 2 patients with angiopulmonography (3.2\%) with stopped bleeding. EBA was done to 28 (44.4\%) patients with COPD complicated by LB. The indication for embolization of the bronchial artery was the detection of direct or indirect signs of bleeding during angiography. In 13 (20.6\%) patients endovascular embolizationwas performed at the "height of bleeding" due to inefficiency of conservative hemostasis. In $5(7.9 \%)$ patients, REO was performed after conservative hemostasis due to the high risk of recurrence of hemorrhage. In 4 (6.4\%) patients with bilateral inflammatory process and unspecified source of pulmonary hemorrhage at bronchoscopy, the technique of delayed embolization of bronchial arteries was used. All patients with unilateral localization of the pathological process after REO achieved a stable hemostatic effect. In the future, $14(22.2 \%)$ patients underwent routine lung surgeries within 4 to 10 days. 12 (19.1\%) patients of the main group were operated on and endovascular occlusion of the bronchial arteries was the main and definitive method of treatment of pulmonary bleeding [27,28]. Mortalityinthe main group was 3.5\% (2 patients with chronic lung abscess, which was complicated by profuse pulmonary hemorrhage.
Patients for temporary hemostasis were performed endovascular embolization of the left bronchial artery at the height of bleeding with subsequent pulmonectomy. Total recurrence of pulmonary hemorrhage was observed in $4(6.4 \%)$ patients. The non effectiveness of conservative therapy in the comparison group served in patients with severe blood loss indications for external surgery - thoracotomy with lobo- or pulmonectomy. Planned surgical interventions were performed in $10(12.8 \%)$ patients, $14(17.9 \%)$ patients were operated on urgent at the height of pulmonary bleeding, and $54(69.2 \%)$ patients were treated only conservatively. Of the 36 patients in the comparison group, 5 (6.4\%) died, with a lethal outcome in the group of conservatively treated outbreaks in $2(3.7 \%)$ patients as a result of recurrent profuse bleeding, and 2 (14.3\%), among planned ones - 1 (10\%) patient. Total recurrence of pulmonary hemorrhagewas noted in $16(29.5 \%)$ patients.

Bronchial arteriography and angiopulmonography are the most reliable methods of detecting the source of pulmonary hemorrhage, and therapeutic embolization of the bronchial arteries is one of the most effective, low-traumatic and reliable methods of stopping pulmonary bleeding. This method can be both independent and final, as well as the stage of preparation of the patient for further radical surgery. However, our studies have shown that endovascular interventions in the area of the bronchial arteries in the period of bleeding, especially profuse, are extremely complex and require careful training both from the operational and technical point of view, and in terms of preparation of the patient.

\section{CONCLUSIONS}

1. In our observations non effective REE of bronchial arteries activity with repeated exposure of LB and hemoptysis observed in $20 \%$ of patients, early in $8.3 \%$ of them. Repeated LB at a later dat. The timing appeared in $13.5 \%$ pain, which required repeated implementation of REEA. CausesofREELBaredifferent. Roentgen-endovascular embolization of bronchial arteries is the method of choice for the treatment of LB and hemoptysis in most patients with $\mathrm{n}$ chronic lung diseases.

2. Bronchial artery angiography gives high efficiency in solving the problem of hemostasis in oncological and nonspecific lung diseases, for determination of localization and source of bleeding. To perform effective endovascular hemostasis; - to gain time for stabilization the patient with the aim of planned surgical treatment.

\section{REFERENCES}

1. Zener R., Bottoni D., Zaleski A., Fortin D., Malthaner R.A., Inculet R.I. (2017). Transarterial embolization of intralobar pulmonary sequestration in a young adult with hemoptysis. J Thorac Dis. 2017; 9 (3): E188-E193. doi: http://doi.org/10.21037/jtd.2017.02.82

2. KimT.E., KwonJ.H., KimJ.S. (2014). Trans-catheter embolization massive hemoptysis from an intralobar pulmonary sequestration: a casereport. Clin Imaging.; 38:326-329. doi: http://doi.org/10.1016/j. clinimag.2012.07.002 
3. Cardenas-Garcia J, Feller-Kopman D. (2018)POINT: Should All Initial Episodes of Hemoptysis Be Evaluated by Bronchoscopy? Yes. Chest 2018; 153:302.doi: http://doi.org/10.1016/j.chest.2017.09.036

4. Mohapatra M., Mishra S., Jena P. (2012). Massive hemoptysis in a case of intralobar pulmonary sequestration associated with pulmonary hypoplasia and meandering right pulmonary vein: diagnosis and management. Case Rep Pulmonol. 2012; Article ID 960948. doi: http:// doi.org/10.1155/2012/960948

5. 0jha V., Samui P.P., Dakshit D. (2015). Role of endovascular embolization in improving the quality of life in a patient suffering from complicated intralobar pulmonary sequestration - a case report. Respir Med Case Rep. 2015; 16:24-28. doi: http://doi.org/10.1016/j.rmcr.2015.02.011 [6] Antonio Borzelli, Andrea Paladini, Francesco Giurazza. (2018). Successful endovascular embolization of an intralobar pulmonary sequestration. Radiol Case Rep. 2018 Feb; 13(1): 125-129. doi: http:// doi.org/ 10.1016/j.radcr.2017.10.003

7. V.Bhargavi,I.Subbanna;Bangalore/IN. (2018). Hemoptysis:an endovascular rescue. ECR 2018. C-1467. doi: http://doi.org/10.1594/ecr2018/

8. Clément Marcelin. (2018). Outcomes of Pulmonary Artery Embolization and Stent Graft Placement for the Treatment of Hemoptysis Caused by Lung Tumor. ECR 2018 / C-1467. doi: http://doi.org/ 10.1183/09031936.001

9. Amar Gupta, Mark Sands, NikunjRashmikant Chauhan. (2018). Massive hemoptysis in pulmonary infections: bronchial artery embolization. European Respiratory Journal 2015 45: 601-603; doi: http://doi. org/ 10.1183/09031936.00199914

10. Fruchter O, Schneer S, Rusanov V, et al. (2015). Bronchial artery embolization for massive hemoptysis: long-term followup. Asian CardiovascThorac Ann 2015;23:55-60 doi: http://doi. org/10.1177/0218492314544310 [12] Ketai LH, Mohammed TL, Kirsch J, et al. (2014). ACR appropriateness criteria ${ }^{\circledast}$ hemoptysis. J Thorac Imaging 2014; 29:W19.doi: http://doi.org/ 10.1097/ RTI.0000000000000084.

11. David R. Sopko, M.D. and Tony P. Smith, M.D. (2011). Bronchial Artery Embolization for Hemoptysis. Semin Intervent Radiol. Mar; 28(1): 48-62. doi: http://doi.org/10.1055/s-0031-1273940

12. Mohapatra M., Mishra S., Jena P. (2012). Massive hemoptysis in a case of intralobar pulmonary sequestration associated with pulmonary hypoplasia and meandering right pulmonary vein: diagnosis and management. Case Rep Pulmonol. 2012; Article ID 960948. doi: http:// doi.org/10.1155/2012/960948

13. Zener R., Bottoni D., Zaleski A., Fortin D., Malthaner R.A., Inculet R.I. (2017). Transarterial embolization of intralobar pulmonary sequestration in a young adult with hemoptysis. J Thorac Dis. 2017; 9 (3) : E188-E193. doi: http://doi.org/10.21037/jtd.2017.02.82

14. Boyko V. V., Ponomarova K. V., Krasnoyarujsky A. G., Korolevska A. Yu., Avdosjev Yu. Our experience of bronchial artery embolization in patients with pulmonary bleeding. Europeansurgery /V. - 60th Annual Meeting of the Austrian Society of Surgery Schnittmengen - Innsbruck, June 19-21.2019.51:72. doi: https://doi.org/10.1007/s10353-019-0600-2

15. Ojha V., Samui P.P., Dakshit D. (2015). Role of endovascular embolization in improving the quality of life in a patient suffering from complicated intralobar pulmonary sequestration - a case report. Respir Med Case Rep. 2015; 16:24-28. doi: http://doi.org/10.1016/j.rmcr.2015.02.011

16. Hosman AE, de Gussem EM, Balemans WAF, et al. (2017). Screening children for pulmonary arteriovenous malformations: evaluation of 18 years of experience. PediatrPulmonol. 2017;52(9):1206-1211. doi: http://doi.org/10.1002/ppul.23704.
17. Antonio Borzelli, Andrea Paladini, Francesco Giurazza. (2018). Successful endovascular embolization of an intralobar pulmonary sequestration. Radiol Case Rep. 2018 Feb; 13(1): 125-129. doi: http:// doi.org/ 10.1016/j.radcr.2017.10.003

18. V. Bhargavi, I. Subbanna; Bangalore/IN. (2018). Hemoptysis: an endovascular rescue. ECR 2018. C - 1467. doi: http://doi.org/10.1594/ ecr2018/C-1467

19. Kateryna Ponomarova (2019). Possibilities of using miniinvasive catheter technologies in the treatment of lung bleeding. ( «EUREKA: Health Sciences» Number 6 (2019), P. 41-48, D0l: 10.21303/25045679.2019.001087.

20. Antonio Borzelli, Andrea Paladini, Francesco Giurazza. (2018). Successful endovascular embolization of an intralobar pulmonary sequestration. Radiol Case Rep. 2018 Feb; 13(1): 125-129. doi: http:// doi.org/ 10.1016/j.radcr.2017.10.003

21. Balch H, Crawford H, McDonald J, O'Hara R, Whitehead K. (2017) Long-term treatment outcomes of embolotherapy in pulmonary arteriovenous malformations in children with hereditary hemorrhagic telangiectasia. Ann Vasc Med Res. 2017;4(4):1064. doi: http://doi. org/10.24296/jomi/249

The topic of GDR surgery of the thoracic cavity of the State Institution "Institute of General and Emergency Surgery. V.T. Zaytseva NAMNU "P.01.14" Development of surgical tactics with the use of mini-invasive technologies in patients with bronchial stump inability ", state registration number 0114U000083. This research became a fragment of this research work.

\section{ORCID and contributorship:}

Kateryna V. Ponomarova - 0000-0001-7327-0632 ${ }^{D}$

Dmitriy V. Minukhin - 0000-0003-3371-1178 ${ }^{\mathrm{A}}$

Denys O. Yevtushenko - 0000-0003-1941-7183 C

Andrey V. Tokarev - 0000-0002-7609-4854 ${ }^{B}$

Oleksandr M. Kudrevych - 0000-0002-2086-8822 ${ }^{E}$

Vasiliy G. Hroma - 0000-0003-1886-3181 ${ }^{F}$

\section{Conflict of interest:}

The Authors declare no conflict of interest.

\section{CORRESPONDING AUTHOR Kateryna V. Ponomarova}

SI V.T. Zaytsev Institute of General and Emergency Surgery of NAMS of Ukraine 1 Balakireva entry, 61103, Kharkiv, Ukraine tel: +380662249679

e-mail:eponkat@gmail.com

Received: 21.02 .2020

Accepted: 06.05 .2020 